\title{
Chances of sperm retrieval during testicular sperm extraction in adult cryptorchid testes
}

\begin{abstract}
Introduction: In this study we investigated the relationship between the preoperative location of the undescended testis, in patients suffering from cryptorchidism, and the presence of sperms in those testis after surgical removal, in adults. We hypothesized that there would be a significant decrease in sperm presence as the anatomical site becomes higher.

Methods: This is a retrospective study (2000-2016) where we used the King Faisal Specialist Hospital and Research Centre electronic patient record database to collect information on adults with cryptorchidism who had undergone orchiectomy or orchiopexy. We divided the patients to three groups according to preoperative anatomical site: abdominal, pelvic and inguinal. We then correlated the site with the surgical biopsy reports with regards to the presence of sperm. We also recorded whether the patients underwent Micro-TESE or random biopsy (TESE).

Results: Our sample was 59 patients aged 15-50 years old with a median of 26 years: 40 were inguinal, 8 were pelvic, and 11 were abdominal. The results showed absence of sperm in all of the patients in the pelvic and the abdominal group. While in the inguinal group, $7 \%$ of these patients had sperm. Furthermore, the biopsies collected from a normal TESE yielded the same result as the samples taken from Micro-TESE in our study.

Conclusion: The chances of successful sperm retrieval were non-existent in all locations except for testis in the inguinal region. The data showed that Micro-TESE, which is far more time consuming, is not superior to random biopsy in sperm extraction.
\end{abstract}

Volume 4 Issue 2 - 2017

\author{
Abdulelah AIGosaibi,' Abdulaziz AIMulhim, ${ }^{2}$ \\ Sultan AIThekafi, ${ }^{3}$ Naif AlHathal ${ }^{3}$ \\ 'College of Medicine, AlFaisal University, Saudi Arabia \\ ${ }^{2}$ College of Medicine, AlFaisal University, Saudi Arabia \\ ${ }^{3}$ Department of Urology, King Saud Medical City, Saudi Arabia
}

Correspondence: Naif AlHathal, Department of Urology, King Faisal Specialist Hospital and Research Centre, Zahrawi Street, Al Maather, Riyadh, Saudi Arabia, Tel 966506104180,

Email nalhathal@kfshrc.edu.sa

Received: October 28, 2016 | Published: February 23, 2017

\section{Introduction}

In addition to an increased risk of developing cancer within the affected testis, ${ }^{1}$ cryptorchidism also has another extremely negative consequence: on fertility., ${ }^{2,3}$ It is known that temperature affects spermatogenesis. $^{4-6}$ Due to the increased temperature of being deeper inside the body, undescended testis will have impaired spermatogenesis, which will lead to reduced and defective sperm formation. $^{7-10}$ In adults presenting with cryptorchidism, they will mostly likely undergo orchiectomy or orchiopexy due to increased risk of malignancy and torsion. ${ }^{11}$ We posed the question: are there any viable sperms in these resected testes? Furthermore, does location of the testis preoperatively have a role in determining the presence of sperm in the resected specimen? Finally, what is the extent of the defect?

As fertility is a major concern in adult males, we feel that this study will help health care professionals offer counseling to their patients that reflects the reality of their chance of fertility. Furthermore, we will investigate whether micro-TESE (microsurgical testicular sperm extraction) has a better chance of sperm extraction from the undescended testes compared to the random biopsy. The significance of this lies in the fact that micro-TESE is extremely time consuming for the surgeon unlike the normal TESE.

\section{Methods}

We retrospectively collected chart information from the King Faisal Specialist Hospital and Research Centre electronic patient record database. Our inclusion criteria were patients with cryptorchidism who had surgical resection of the testes within the past 16 years (2000/2016) and also had preoperative location of the testes and biopsy results available. We reviewed their surgical reports for biopsy results regarding sperm presence as well as preoperative site of cryptorchidism. Furthermore, we noted which patients underwent Micro-TESE (microsurgical testicular sperm extraction.) The sperm presence as treated as binary data (has sperm, does not have sperm). The site was one of 3 possible sites: inguinal, pelvic or abdominal. Patients who lacked complete information regarding postsurgical biopsy or pre-operative location of the testis were excluded. The two main variables in the study were the location of the testis before surgery and the presence of sperms in them in the post-op analysis. Other data collected was the date of surgery, MRN, age at procedure, semen analysis, hormone profile, imaging modality for locating the testis prior to surgery, size and side, surgery type, and histopathology results. The information was correlated and checked for any significant relationship between the main variables and as well as their relationship to the secondary variables. 


\section{Results}

Of the charts reviewed, 95 patients underwent orchiectomy or orchiopexy due to cryptorchidism. 35 patients were excluded due to incomplete data. Of the 59 patients, the undescended testes were in the inguinal region in 40 patients, in the pelvic region in 8 patients, and in the abdominal region in 11 patients. Biopsies of those with the testes in the abdominal and pelvic region showed no sperms at all (Table 1). 4 out of the $40(\sim 10 \%)$ patients with the testes in the inguinal region showed the presence of viable sperms (Table 2). In addition, ten patients underwent micro-TESE, of these patients, $1(10 \%)$ had viable sperms as opposed to the 3 out of 30 patients $(\sim 10 \%)$ sperm presence in those who underwent random biopsy.
Table I Patient demographics average

\begin{tabular}{ll}
\hline Age at procedure & $\mathbf{2 5 . 7} \pm \mathbf{7 . 3}$ \\
\hline FSH & $18.7 \pm 16.7$ \\
LH & $11.4 \pm 8.1$ \\
Prolactin & $13.6 \pm 8.7$ \\
Testosterone & $15.0 \pm 8.1$ \\
TSH & $3.5 \pm 3.3$ \\
\hline
\end{tabular}

Table 2 Presence of viable sperms Vs Testis location

\begin{tabular}{llll}
\hline Location & Number of patients $(\mathbf{n}=\mathbf{5 9})$ & Number of patients with viable sperms & Frequency of sperm presence \\
\hline Inguinal & 40 & 4 & $10 \%$ \\
Pelvic & 8 & 0 & $0 \%$ \\
Abdominal & II & 0 & $0 \%$ \\
\hline
\end{tabular}

\section{Discussion}

The results showed that location does indeed affect the presence of viable sperms in the undescended testes and that having them in the inguinal region yields the greatest chance of having viable sperms. What is actually significant in practice would be the fact that those who had the undescended testis in both the abdomen and pelvis had a zero chance of having sperms. This information is crucial for the healthcare provider when counseling these kinds of patients. Another significant finding in this study was the unremarkable difference between the use of random biopsy (TESE) and Micro-TESE ${ }^{12}$ as it yielded the same results in all patients that underwent the sperm extraction techniques. Hinting towards the absence of superiority of Micro-TESE in these patients, which needs to be further investigated using a larger sample size in future research.

In a research done in Turkey, Ateş F et al. ${ }^{13}$ on 244 adult unilateral cryptorchid patients, 4 patients whose pre-op surgical site was inguinal were found to have hypospermatogenesis (viable sperm) as opposed to abdominal patients who all had either sertoli cell syndrome, testicular atrophy or maturation arrest. This correlates with the data we have. Study Bernie, Aaron M et al. ${ }^{14}$ is a large scale meta analysis which showed that Micro-TESE was the most superior method of sperm extraction compared to conventional TESE. The findings of this meta-analysis do not correlate with the findings in our study. This information will contribute to the healthcare provider's ability to council patients on their chances of having a child and. ${ }^{15}$

\section{Conclusion}

The data has supported out hypothesis that higher anatomical negatively impacts success rates of sperm extraction. Furthermore, contrary to previous studies, Micro-TESE has failed to prove more successful than random biopsy in sperm extraction. We feel that more research should be done on this topic and with a larger sample size. Furthermore, the impact of using micro-TESE for patients with cryptorchidism should also be further evaluated.

\section{Acknowledgement}

None.

\section{Conflicts of interest}

The author declares there is no conflict of interest.

\section{References}

1. National Kidney Foundation. K/DOQI clinical practice guidelines for chronic kidney disease: evaluation, classification, and stratification. $\mathrm{Am}$ J Kidney Dis. 2002;39(2 Suppl 1):S1-S266.

2. Ferguson L, Agoulnik AI. Testicular Cancer and Cryptorchidism. Front Endocrinol (Lausanne) doi:10.3389/fendo.2013.00032.

3. Schärli AF. Cryptorchidism and infertility. Pediatric Surgery International. 1998;14(1-2):1-1.

4. Kumar V, Cotran RS, Robbins SL. Robbins basic pathology. Philadelphia, PA: Saunders, Turk J Uro. 2013;42(2):74-79.

5. Robinson D, Rock J, Menkin MF. Control of Human Spermatogenesis Intrascrotal Temperature. JAMA. 1968;204(4):290-297.

6. Lania C, Grasso M, Mantovani G, et al. Cryptorchidism: Review and Evaluation of the Fertility of Adult Cryptorchids. Journal of Andrology. 1984;5(S2):125-127.

7. Guyton AC, Hall JE. Textbook of medical physiology. Philadelphia: Saunders, USA. 2000.

8. 1981 Cryptorchidism and Fertility. N Engl J Med. 2002;304(3):173-173.

9. van Brakel J, Kranse R, de Muinck Keizer-Schrama SM, et al. Fertility potential in men with a history of congenital undescended testes: A longterm follow-up study. Andrology. 2012;1(1):100-108.

10. Huff DS, Hadziselimovic F, Snyder HM, et al. Histologic maldevelopment of unilaterally cryptorchid testes and their descended partners. Eur $J$ Pediatr. 1993;152(S2):S11-S14.

11. Moretti E, Di Cairano G, Capitani S, et al. Cryptorchidism and Semen Quality: A TEM and Molecular Study. J Androl. 2006;28(1):194-199.

12. Rogers E, Teahan S, Gallagher H, et al. The Role Of Orchiectomy In The Management Of Postpubertal Cryptorchidism. J Urol. 1998;159(3):851854.

13. van Brakel J, Kranse R, de Muinck Keizer-Schrama SM, et al. Fertility Potential in a Cohort of 65 Men with Previously Acquired Undescended Testes. J Pediatr Surg. 2014;49(4):599-605. 
14. Ateş F, Soydan H, Okçelik S, et al. Clinical and histopathological results of the adult patients with unilateral cryptorchidism. Turk $J$ Urol. 2016;42(2):74-79.
15. Bernie AM, Mata DA, Ramasamy R, et al. Comparison of microdissection testicular sperm extraction, conventional testicular sperm extraction, and testicular sperm aspiration for nonobstructive azoospermia: a systematic review and meta-analysis. Fertil Steril. 2015;104(5):1099-1103. 